\title{
Experimental validation of depletion calculations with VESTA 2.1.5 using JEFF-3.2
}

\author{
Wim Haeck ${ }^{\mathrm{a}}$ and Raphaëlle Ichou \\ Institut de Radioprotection et de Sûreté Nucléaire (IRSN), PSN-EXP/SNC, 92260 Fontenay-aux-Roses, France
}

\begin{abstract}
The removal of decay heat is a significant safety concern in nuclear engineering for the operation of a nuclear reactor both in normal and accidental conditions and for intermediate and long term waste storage facilities. The correct evaluation of the decay heat produced by an irradiated material requires first of all the calculation of the composition of the irradiated material by depletion codes such as VESTA 2.1, currently under development at IRSN in France. A set of PWR assembly decay heat measurements performed by the Swedish Central Interim Storage Facility (CLAB) located in Oskarshamm (Sweden) have been calculated using different nuclear data libraries: ENDF/B-VII.0, JEFF-3.1, JEFF-3.2 and JEFF-3.3T1. Using these nuclear data libraries, VESTA 2.1 calculates the assembly decay heat for almost all cases within $4 \%$ of the measured decay heat. On average, the ENDF/B-VII.0 calculated decay heat values appear to give a systematic underestimation of only $0.5 \%$. When using the JEFF-3.1 library, this results a systematic underestimation of about $2 \%$. By switching to the JEFF-3.2 library, this systematic underestimation is improved slighty (up to $1.5 \%$ ). The changes made in the JEFF-3.3T1 beta library appear to be overcorrecting, as the systematic underestimation is transformed into a systematic overestimation of about $1.5 \%$.
\end{abstract}

\section{Introduction}

Isotopic characterisation of irradiated fuel via depletion and decay calculations is essential in reactor core neutronics and safety calculations. This is especially important for the calculation of the temporal evolution of the power distribution and the reactor kinetics parameters but also for the evaluation of the residual decay heat produced by the fuel assemblies in the core of a nuclear reactor as a function of time.

The removal of decay heat is a significant safety concern in nuclear engineering both for normal reactor operation and for intermediate and long term waste storage facilities. The decay heat removal systems that have to be put in place to ensure the removal of the decay heat depend largely on the time scale in which the decay heat is produced. Directly after the shutdown of a reactor (both in normal or accidental conditions), the decay heat represents about $6 \%$ of the original reactor power. A week later, this has already dropped to $0.2 \%$ of the original reactor power. Even after 20 years of cooling an average fuel assembly will still produce a few hundred Watt of decay heat.

The correct evaluation of the decay heat produced by an irradiated material requires first of all the calculation of the composition of the irradiated material, from which we can derive the decay heat $P$ (in $\mathrm{W} \cdot \mathrm{gHM}^{-1}$ ) as follows:

$$
P=\sum_{i} Q_{i, \text { ave }} \frac{\ln 2}{T_{i, 1 / 2}} N_{i}
$$

\footnotetext{
a e-mail: wim.haeck@irsn.fr
}

with:

- $Q_{i \text {,ave }}$ the average decay energy released per desintegration of nuclide $i$ given in Joule (this data can be found in the radioactive decay data);

- $T_{i, 1 / 2}$ the half life of nuclide $i$ given in seconds;

- $N_{i}$ the concentration of nuclide $i$ for the irradiated material in atoms. $\mathrm{gHM}^{-1}$.

For depletion codes such as VESTA [1] that are used for decay heat estimations, experimental validation is paramount. Over the last couple of years, IRSN has established an important experimental validation database using a set of 76 samples of radiochemical assay data and decay heat measurements [2] for a wide variety of reactor and fuel types (PWR, VVER, MOX, $\mathrm{UO}_{2}, \mathrm{UO}_{2}-\mathrm{Gd}_{2} \mathrm{O}_{3}$ fuel).

The results of the calculations are definetely influenced by the underlying nuclear data library [3]. We will further illustrate this aspect in this paper by comparing the results for the decay heat measurements from the VESTA experimental validation database using four nuclear data libraries, namely JEFF-3.1, JEFF3.2, JEFF-3.3T1 and ENDF/B-VII.0. The experiments that have been selected for this work are PWR assembly decay heat measurements performed by the Swedish Central Interim Storage Facility (CLAB) located in Oskarshamm (Sweden) [4].

\section{Overview of experimental data}

The CLAB measurements were performed on a total of 39 BWR assemblies and 34 PWR assemblies from various reactors in Sweden. The PWR assembly measurements come from the Ringhals 2 unit (23 measurements for $1815 \times 15$ assemblies) and the Ringhals 3 unit

(C) The Authors, published by EDP Sciences. This is an Open Access article distributed under the terms of the Creative Commons Attribution License 4.0 (http://creativecommons.org/licenses/by/4.0/). 
(20 measurements for $1617 \times 17$ assemblies). All assemblies are $\mathrm{UO}_{2}$ assemblies covering a ${ }^{235} \mathrm{U}$ enrichment range of 2.1 to $3.4 \%$, burn up range of 20 to 51 $\mathrm{MWd} \cdot \mathrm{kgHM}^{-1}$ for cooling times from 13 to 23 years.

These CLAB measurements were performed using a pool calorimeter located underwater in the spent fuel storage pool. It is $4.6 \mathrm{~m}$ long and consists of two concentric carbon steel pipes separated by urethane foam insulation to reduce heat loss to the pool. A so called static mode is the measurement method in which the water temperature increase in the vessel is measured. The decay heat of the assembly is then determined by calibrating the temperature rise against an electric heater assembly. The target accuracy for the calorimeter was about $2 \%$ (confidence interval of 95\%).

\section{Decay heat calculation procedure}

For this work, the VESTA 2.1.5 calculations were performed using MCNPX 2.6.0 [5] and PHOENIX (the built in depletion module of VESTA 2.1.5). All fuel assemblies have been modelled in $2 \mathrm{D}$, which is a common way of modelling PWR assemblies in deterministic codes. The geometrical model used also takes advantage of symmetry as much as possible. Surrounding assemblies were not included in the models. For the depletion calculations, each fuel pin and guide tube have been considered as separate depletion zones. No radial zones are considered in the fuel pins or absorber rods (for the Ringhals 3 assemblies). The spacer grids used in the various assemblies were not taken into account in the assembly model and will therefore not contribute to the final decay heat calculation.

The number of particles used in each transport calculation are chosen so that the statistical total flux errors are lower than $0.1 \%$ for every fuel pin in the calculation. This is done to reduce the statistical fluctuation on the final $\mathrm{C} / \mathrm{E}$ values. The water moderator in these models contains an average boron concentration. The moderator and fuel pin cladding temperatures are set to $600 \mathrm{~K}$ and the fuel temperature at $900 \mathrm{~K}$ which is close to the average fuel temperature over the entire irradiation history for all assemblies. No temperature evolution as a function of time has been modelled. All these simplifications can potentially introduce a small additional uncertainty on the calculation results.

The irradiation history for each measured assembly was divided into a number of steps corresponding to an average burn up of $1 \mathrm{MWd} \cdot \mathrm{kgHM}^{-1}$ in every step. In addition, no predictor-corrector algorithm is used over the time steps.

\section{Nuclear data libraries}

All nuclear data files used by VESTA (and the underlying transport codes such as MCNP) have been produced using the NJOY nuclear data processing system [6] through the GAIA software [7]. GAIA is a computer software developed at IRSN to minimise the effort on the generation of nuclear data libraries using NJOY and provide additional quality assurance. The library files produced consist of ACE files (which will only be used by MCNP or MCNPX) and PENDF files (which will only be used by VESTA). GAIA not only automates the entire
NJOY processing path, but it will also perform a number of additional operations to verify the produced files and add any missing data required for the applications in question.

The software generates a tailormade NJOY input file for each nuclide without intervention of the user using data found in the nuclide's ENDF file (such as the initial temperature of the ENDF file, whether or not the nuclide is fissile, whether or not it has unresolved resonances, etc.). When the library files have been generated, GAIA will also process the output from NJOY and extract all messages and warnings from NJOY that are deemed important. A print out including a short explanation of the messages in question is then printed to the listing (the user can, if needed, override this setting to extract all messages and errors regardless of their importance). The most important test provided by the software is the verification of the unresolved resonance probability tables. GAIA will check for the presence of any negative cross section values in the tables. If such negative values are detected, GAIA will rerun NJOY but omit the generation of the unresolved resonance probability tables (no selfshielding calculations using probability tables in the unresolved resonance range can thus be performed).

Once the library files pass the NJOY processsing and verification stage, GAIA will add data from the original ENDF file to the final PENDF file for use by VESTA. This includes the average number of neutrons per fission, the components of energy release per fission and the isomeric branching ratio data.

In order to obtain a consistent nuclear data library for depletion applications, we still need to add the neutron induced fission yield and decay data libraries (which are stored as separate ENDF files within the VESTA libraries). The JEFF-3.1 and ENDF/B-VII.0 library uses their respective fission yield and decay data files. Since the JEFF-3.2 and JEFF-3.3T1 evaluations did not include any fission yield or decay data, the data from JEFF-3.1.1 was use instead.

\section{Results}

Figure 1 gives the results for these assembly decay heat calculations. The top graph gives a graphical representation of the calculated to experimental $(\mathrm{C} / \mathrm{E})$ value while the bottom graph gives the difference between the calculated and experimental value with respect to the experimental error $\sigma$. The grey area in these bottom graphs corresponds to an uncertainty range of two standard deviations. These results are given as a function of the measured decay heat. The red dots correspond to the Ringhals 2 measurements and the blue triangles correspond to the Ringhals 3 measurements.

As expected, we see that the various nuclear data libraries give slightly different results. Overall, it is difficult to actually say which library performs the best because of multiple reasons. First of all, there are the modelling approximations that are introduced which can bias the results. Secondly, the uncertainty on the decay heat measurements is of the order of a couple of percent which is roughly the same as the spread on the average differences observed between the libraries.

We can see in Fig. 1 that we obtain the "best" results using the ENDF/B-VII.0 library. In this case, the decay heat values for almost all assemblies are calculated within 


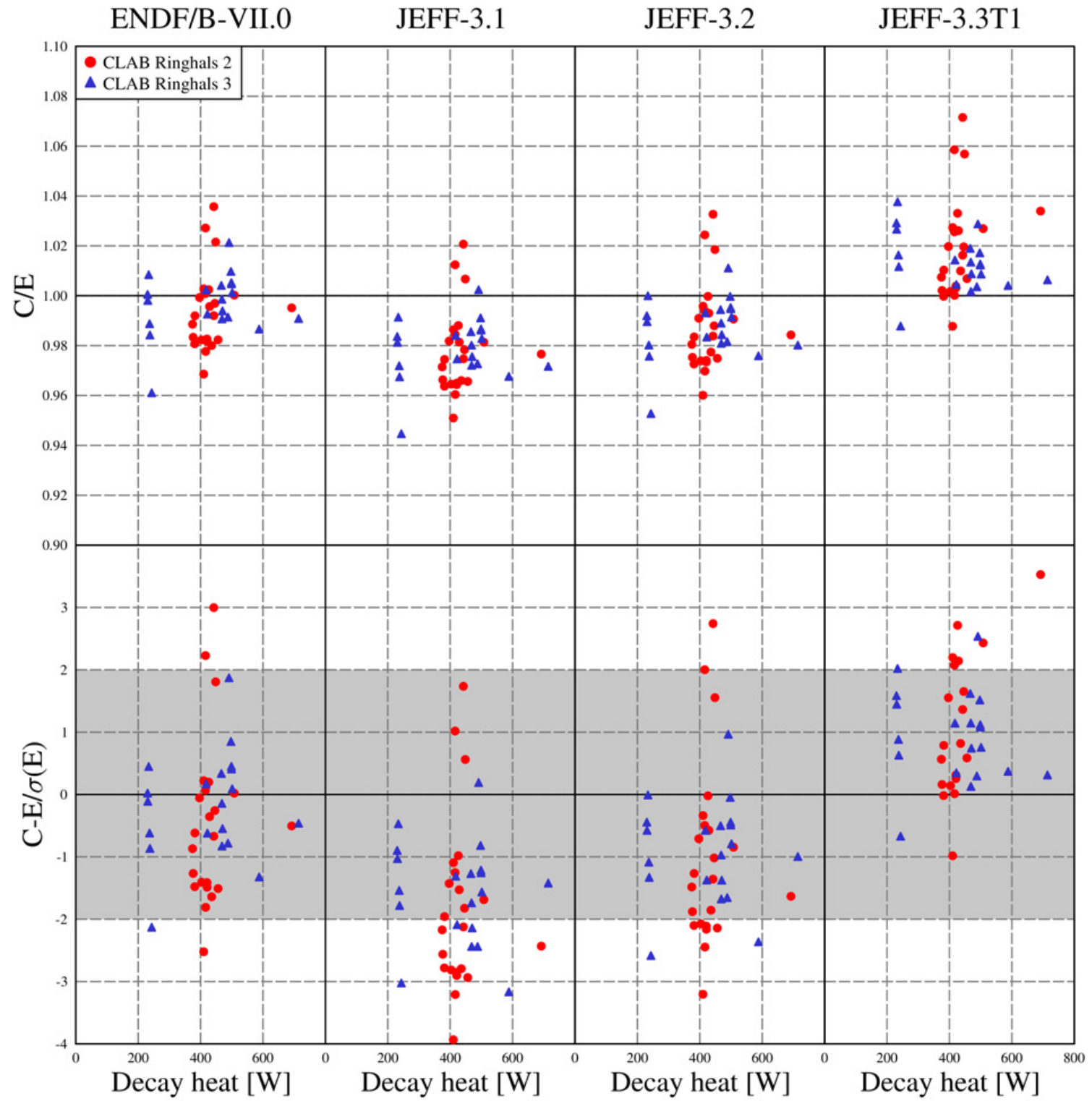

Figure 1. C/E results (top) and C-E/ $\sigma(E)$ (bottom) for the assembly decay heat calculations for the ENDF/B-VII.0, JEFF-3.1, JEFF-3.2 and JEFF-3.3T1 nuclear data libraries as a function of measured decay heat. The grey area in the bottom graphs corresponds to an uncertainty range of two standard deviations.

$2 \%$ of the experimental value. The average $\mathrm{C} / \mathrm{E}$ value for this library also exhibits a very small underestimation (of the order of $0.5 \%$ ). This fact is confirmed in the bottom graph for ENDF/B-VII.0 which shows that almost all calculated values lie within two standard deviations of the corresponding experimental value.

The results obtained using the different versions of the JEFF library are also quite interesting. The oldest version (JEFF-3.1) gives us decay heat values within $4 \%$ of the measured values and on average $1.8 \%$ below the ENDF/BVII.0 values. From an average point of view, this library appears to underestimate the decay heat values as the average $\mathrm{C} / \mathrm{E}$ value exhibits an underestimation of about $2 \%$ which is confirmed in the bottom graph as about $1 / 3$ of all points lie outside of two standard deviations of the experimental value.

Switching to the JEFF-3.2 or JEFF-3.3T1 library changes this result. The change to JEFF-3.2 reduces the underestimation we observed by half to about $1 \%$ resulting in decay heat values that differ little from the values obtained with the ENDF/B-VII.0 library (the JEFF-3.2 values lie only $0.8 \%$ below those of ENDF/B-VII.0).

The use of the JEFF-3.3T1 beta library results in a reversal of this situation. Where JEFF-3.1 and JEFF-3.2 gave decay heat values under the ENDF/B-VII.0 results, the JEFF-3.3T1 library gives decay heat values about $1.5 \%$ higher than ENDF/B-VII.0. With respect to the JEFF-3.2 library, the decay heat values increase by about $2.5 \%$. It is interesting to note that this change is only due to changes in the neutron reaction data as both the JEFF-3.2 and JEFF3.3T1 library have the same fission yield and decay data (taken from JEFF-3.1.1).

\section{Conclusions}

Isotopic characterisation of irradiated fuel via depletion and decay calculations is essential in reactor core neutronics and safety calculations. This is especially 
important for the calculation of the temporal evolution of the power distribution and the reactor kinetics parameters but also for the evaluation of the residual decay heat produced by the fuel assemblies in the core of a nuclear reactor as a function of time. Results of these parameters have various sources of uncertainty. In this paper, we have illustrated the influence of the nuclear data library as a whole on the calculation of decay heat. Results for the decay heat calculations using four nuclear data libraries (JEFF-3.1, JEFF3.2, JEFF-3.3T1 and ENDF/B-VII.0) have been compared using the CLAB assembly decay heat measurements used in the experimental validation of VESTA.

Using these nuclear data libraries, VESTA 2.1 calculates the assembly decay heat for almost all cases within $4 \%$ of the measured decay heat. On average, the ENDF/B-VII.0 calculated decay heat values appear to give a systematic underestimation of only $0.5 \%$. When using the JEFF-3.1 library, this results in a systematic underestimation of about $2 \%$. By switching to the JEFF3.2 library, this systematic underestimation is improved slighty (up to $1.5 \%$ ).

The changes made in the JEFF-3.3T1 beta library appear to be overcorrecting, as the systematic underestimation is transformed into a systematic overestimation of about $1.5 \%$. The change from JEFF-3.2 to JEFF-3.3T1 also increases the decay heat values by about $2.5 \%$ and this change can be contributed to the neutron induced reaction data because the fission yield and decay data are actually the same for these two nuclear data libraries.
It is difficult to judge which library gives the best results because the differences between the libraries (of the order of 2 to 4 percent) are of the same order of magnitude as the experimental error on the decay heat measurements.

\section{References}

[1] W. Haeck, VESTA User's Manual - Version 2.1.0, IRSN Report DSU/SEC/T/2012-081 Index A (IRSN, France, 2012)

[2] W. Haeck, Experimental validation of VESTA 2.1.5 Volume 1: Methodology and overview of results, IRSN Report PSN-EXP/SNC/2014-237 Index A (IRSN, France 2014)

[3] W. Haeck, R. Ichou, F. Ecrabet, J. Dannet, Experimental validation of decay heat calculations using VESTA 2.1, PHYSOR 2014 (Kyoto, Japan, 2014)

[4] F. Sturek, L. Agrenius, Measurements of decay heat in spent nuclear fuel at the Swedish interim storage facility CLAB, SKB Rapport R-05-62 (Svensk Kärnbränslehantering AB, Sweden, 2006)

[5] J.S. Hendricks et al., MCNPX, VERSION 2.6.a, LAUR-05-8225 (Los Alamos National Laboratory, USA, 2005)

[6] R.E. MacFarlane, D.W. Muir, The NJOY Nuclear Data Processing System Version 91, LA-12470-M (Los Alamos National Laboratory, USA, 1994)

[7] W. Haeck, GAIA User's Manual - Version 1.0.0, IRSN Report PSN-EXP/SNC/2015-165 Index A (IRSN, France, 2015) 